\title{
El tipo de cambio en el Perú
}

\author{
Econ. Juan MORALES ROMERO
}

\section{RESUMEN}

El presente artículo tiene como objetivo explicar las razones por lo que el precio del dólar aumenta o disminuye en el mercado cambiario es decir experimenta fluctuaciones, que puedan afectar a otros mercados. Ante los cambios bruscos o volatilidad del tipo de cambio, el banco central interviene en el mercado de dólar comprando o vendiendo dólares a fin de reducir la volatilidad del tipo de cambio, dichas intervenciones el BCR lo realiza bajo un contexto de sistema de tipo de cambio flexible en el que adopta o bien la flotación limpia (No intervención del Banco Central en el mercado cambiario) o flotación administrada o sucia (Intervención del Banco Central en el mercado cambiario).

\begin{abstract}
The present article aims to explain the reasons why the dollar exchange rate increase or decrease in the exchange market that is to say experiencing fluctuations, which may affect other markets. Because of these variations or volatility in the exchange rate, the central bank intervenes in the market by buying or selling dollars dollar to reduce the volatility in the exchange rate, such interventions of the Central Bank is performed by applying a flexible exchange rate in which adopts "clean float" (no central bank intervention in the exchange market) or "managed or dirty float" (Central Bank intervention in the foreign exchange market).

El Banco central interviene en el mercado cambiario comprando o vendiendo dólares según la tendencia del dólar a la baja o al alza. En tal sentido el Banco Central interviene en el mercado cambiario comprando dólares cuando el tipo de cambio experimenta una caída o tendencia bajista con volatilidad, el propósito de dicha intervención es incrementar la demanda de dólares a fin de que el tipo de cambio se incremente y con ello evitar la caída del dólar, sin embargo el BCR para comprar dólares tiene que emitir soles para comprar los dólares ampliando la emisión de dinero ( expansión de la base monetaria o emisión primaria) que debe ser esterilizada a fin de evitar que genere presiones inflacionarias. La otra forma en que interviene el BCR en el mercado cambiario es vendiendo dólares a fin de evitar una tendencia alcista del tipo de cambio con volatilidad siendo el objetivo de dicha intervención incrementar la oferta de dólares con el objetivo
\end{abstract}


de que el precio del dólar en términos de soles experimente una reducción de su precio, la consecuencia de la venta de dólares por parte del BCR es la perdida de divisas del BCR o caída de las Reservas Internacionales del Banco Central. Es importante señalar que el dólar experimenta tendencia a la baja cuando se reduce la demanda de dólares ( menor compra de dólares por los agentes económicos) y por el lado de la oferta el tipo de cambio experimenta una caída cuando aumenta la oferta de dólares de la economía peruana, dicha situación ocurre cuando ingresan dólares a la economía peruana producto de nuestros superávits comerciales, el mayor rendimiento bursátil de la BVL, una mayor tasa de interés de política monetaria del BCR respecto a la tasa de interés de política monetaria de un banco central del resto del mundo, menor riesgo país, y las expansión monetarias de EE.UU denominados estímulos monetarios de la Reserva Federal de EE.UU que se le conocen como política monetaria no convencional) estímulo monetario que provocan una migración de dólares de EE.UU a las economías del Resto del mundo que incrementan su oferta dólares y provocan la caída de su tipo de cambio.

De otro lado el dólar experimenta una tendencia al alza cuando se incrementa la demanda de dólares (mayor compra de dólares por los agentes económicos) y por razones de oferta el tipo de cambio observa tendencia alcista cuando se contrae la oferta de dólares debido a la salida de dólares de la economía peruana que se explican por los déficits comerciales, menor rendimiento en la plaza bursátil limeña respecto a las bolsas de valores del resto del mundo, mayor riesgo país y también por el retiro del estímulo monetaria de la Reserva Federal de EE.UU entre otras variables.

En año 2013 el dólar observó dos comportamientos diferenciados, en el primer semestre del año una depreciación del dólar o debilitamiento de la divisa extranjera ( caída en el precio del dólar en términos del Nuevo Sol) - apreciación de la moneda local y en el segundo semestre de 2013 una fuerte volatilidad, producto de las marchas y contramarchas del presidente de la Reserva Federal de EE.UU Ben Bernake respecto al retiro o continuación del estímulo monetario de la FED denominado flexibilización cuantitativa o política monetaria no convencional, que se adopta cuando la tasa de interés de instrumento de política monetaria ya está bordeando el $0 \%$.

Si bien es cierto el comportamiento del dólar debe de obedecer a razones fundamentales que las determina el mercado, es decir la demanda y oferta de dólares, los factores que explicaron la evolución del dólar corresponde a los efectos de las decisiones de política monetaria adoptada por las economías desarrolladas específicamente la de EE.UU.

En el primer semestre del año 2013, el debilitamiento de las economías de Europa, EE.UU y la desaceleración de China contrajeron nuestra demanda externa 
(demanda de exportaciones de productos peruanos por el resto del mundo) y con ello una reducción del superávit comercial que tuvo un efecto marginal en el dólar, pero la variable que contribuyó muy significativamente en la caída del dólar o apreciación del sol en nuestra economía fue la política monetaria expansiva de la FED de EE.UU aplicada para estimular a la debilitada economía norteamericana, el estímulo monetario aplicado por el Banco Central de EE.UU fue de carácter no convencional es decir no se utilizó como instrumento la tasa de política monetaria de la Reserva Federal sino se optó por inyecciones muy importantes de dólares a la economía de EE.UU, que aumento la oferta de dinero de EE.UU para inducir a un aumento de la inversión y el consumo. Sin embargo un importante porcentaje de la inyección de dólares de la FED se trasladaron a las economías de América Latina en busca de rentabilidades provocando que la oferta de dólares en el caso específico del Perú, se incremente y con ello la mayor oferta de dólares genere un descenso en el precio del dólar, situación que persistió en la primera parte del año y que generó una significativa intervención del Banco Central de Reserva del Perú en el mercado cambiario comprando dólares a fin de inducir a un mayor aumento de la demanda de dólares con el propósito de atenuar la caída del tipo de cambio. Sin embargo la intervención activa del BCRP en el mercado cambiario comprando dólares se realizan con soles que emite el BCR que esperemos hayan sido neutralizados a través de operaciones de esterilización para evitar algún tipo de presión inflacionaria.

En la segunda parte del año 2013, se observa una desaceleración económica reflejada en menores tasas de crecimiento positiva del PBI explicada por la reducción de la demanda externa cuyo comportamiento derivó en déficit comercial, producto de la salida de dólares para pagar el exceso de importación de bienes. La salida de dólares tuvo como consecuencia la disminución de la oferta de dólares que presionó al incremento en el precio de la divisa extranjera. Sin embargo en este periodo el dólar experimentó una volatilidad al alza por el anuncio de la Banca Central de EE.UU ( Reserva Federal - FED ) de retirar el estímulo monetario debido a que la economía de EE.UU ya daba señales de fortalecimiento, los mercados adoptaron el anuncio de la FED con mucho nerviosismo, que dio origen a una mayor incertidumbre en los mercados cambiarios del mundo de la que no escapo el Perú, frente a dicha noticia el dólar subió en el Perú por lo que el BCRP, ya no salió a comprar dólares sino a venderlos para evitar mayores alzas en el precio del dólar.

Se espera que el retiro del estímulo monetario se realice a lo largo del 2014 por lo que se proyecta que el dólar en el Perú empezará a experimentar incrementos en su precio, es decir el tipo de cambio aumentará. De tal manera si el ritmo de subida del dólar es muy brusco, observaremos con mayor frecuencia intervenciones del BCR vendiendo dólares que se inyectan a la economía a fin de aumentar la oferta de dólares para compensar la salida de capitales a EE.UU producto del retiro del estímulo monetario de la FED. 
Es importante recordar, que las intervenciones del BCRP en el mercado cambiario se realizan comprando o vendiendo dólares y su intervención es en un contexto donde el dólar experimenta cambios bruscos o volatilidad.

En el primer caso el BCRP compra dólares cuando observa una caída brusca del tipo de cambio a fin de atenuar la disminución del precio de la divisa extranjera. Las razones que explican la disminución del tipo de cambio son una mayor oferta de dólares debido a un mayor ingreso de capitales de corto plazo denominados capitales golondrinos o especulativos, que solo buscan redituar en el corto plazo y los dólares que provienen de la economía de EE.UU por el estímulo monetario aplicado por la FED.

En tanto el BCRP vende dólares ante incrementos significativos en el precio del dólar tal como ocurrió con el anuncio por parte de la FED de retirar gradualmente el estímulo monetario. Las razones que explican un aumento en el precio del dólar son una contracción de la oferta de dólares o una mayor demanda de dólares por parte de los agentes económicos.

Una conclusión importante es que el comportamiento a la baja y a la alza del tipo de cambio en el mercado cambiario obedeció en forma significativa al comportamiento del entorno económico externo, específicamente de EE.UU, que en lo que va del año 2014 ya empezó a retirar en forma gradual su estímulo monetario que incidirá en el mediano plazo a un mayor precio del dólar.

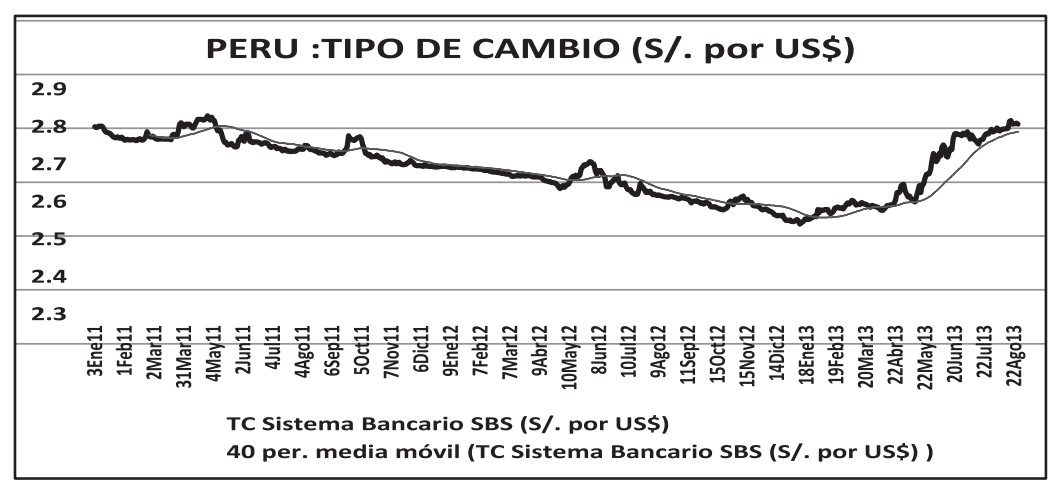

Fuente: Banco Central de Reserva del Perú

Elaboración: Departamento de Ciencias Económicas de la FACEE

\section{BIBLIOGRAFÍA}

1. Morales Romero, Juan (2007). La desdolarización le da paso a la solarización. Diario Expreso.

2. Gonzalez Izquierdo, Jorge (2013). Teoría y Política Económica-La experiencia peruana. Universidad del Pacifico. 